\title{
Capacitive Imaging of Impact Damage in Composite Material
}

Maxim Morozov, William Jackson and S.G.Pierce

Department of Electronic and Electrical Engineering, University of Strathclyde

Technology \& Innovation Centre, 99 George Street, Glasgow, G1 1RD, UK

$+44(0) 1414447406$

Maxim.Morozov@strath.ac.uk

\begin{abstract}
This work presents capacitive non-destructive imaging of impact damage in woven fibre CFRP using a coplanar capacitive sensor. Novelty of the approach consists in that spectral characteristics of the capacitive sensor response to CFRP properties are firstly used to establish excitation frequencies for optimum imaging of delamination. For the tested samples optimum excitation frequencies were found to be above 200MHz. Clear capacitive images of delaminations were obtained on each sample. Ultrasonic (UT) Cscans of delaminations due to impacts were acquired using phased array technique to confirm presence and extent of the delaminations. Capacitive imaging technique can be used as complementary to UT for imaging shallow defects in composite materials at depths where UT has insufficient time domain resolution.
\end{abstract}

Keywords. Carbon fibre, delamination, non-destructive testing, capacitive imaging.

\section{Introduction}

Composite laminated materials find increasing use in modern engineering structures, such as wind turbine blades and especially airframes, due to their high specific strength and stiffness. However, such materials, e.g. carbon fibre-reinforced plastics (CFRP), are 
brittle and have no significant strength in the thickness direction. Therefore, composite laminates are particularly susceptible to damage caused by transverse impacts [1]. The common damage modes inside the panel are delamination (separation of plies), matrix cracking, and fibre breakages [2]. A delamination is a crack which runs in the resin component (approximately $0.0007 \mathrm{~mm}$ in graphite/epoxy laminates) between plies of different fibre orientation and not between lamina in the same ply group [3].

Upon low or intermediate velocity impacts the damage inside the target panel could be large and the laminated composite panel can suffer a significant loss of its designed strength sometimes without a visible indication of damage on the impacted surface [4], thus this is considered to be the most detrimental damage; the impact damage being difficult to detect and often leading to a serious reduction of the structural integrity and mechanical properties [5]. Inter-laminar stresses or mismatching of bending stiffness between two adjacent laminate have been suggested as causes of delamination in the composite laminates subjected to low velocity impact [6] and it has been reported that the apparent damaged area increases monotonically with impact energy [7]. Tolerance to damage is an important requirement in the design and fabrication of composite structural components. The ability of a component to contain a flaw of a given size without serious loss of its structural integrity is of prime concern [1]. Since the full extent of damage due to low-velocity impacts in composite structures is not generally visible to the unaided eye, Non-Destructive Testing and Evaluation (NDT\&E) methods are needed for delamination detection and quantification [8].

A comprehensive overview of existing methods of non-destructive evaluation of polymer matrix composites is given in [9]. NDT\&E methods for composite materials can roughly be classified according to the underlying physical principles as follows: visual, acoustic/ultrasonic, electromagnetic (inductive, capacitive, microwave and thermography), radiographic (X-rays, gamma-rays) [9]. It has been pointed out above 
that visual methods are of limited benefit for low energy impact damage detection as a flaw produced, if any, is smaller than the subsurface delamination. Radiographic testing methods involve radiation hazard. Moreover, delaminations and planar cracks are difficult to detect using radiography. Ultrasonic Testing (UT), particularly by means of Phased Arrays (PA), naturally offers itself for detecting discontinuities such as delaminations since these represent effective reflectors of ultrasonic waves [10-12]. Minor limitation of PA UT might be related to the requirement to have an acoustic couplant, in many cases water, which adds cost and complexity to the inspection procedure or might be undesirable altogether depending on the structure to be inspected. Use of guided wave imaging to detect hidden delamination in multi-layer composites has been presented in [13-16] and Lamb waves have been successfully demonstrated for the case of internal delaminations initiated by low-velocity impacts [17]. Additional ultrasonics techniques include noncontact laser ultrasonic imaging which has been deployed for detection and visualization of delaminations, debonding as well as porosity $[14,18,19]$. The limitation of ultrasound NDT when testing thin layered structures arises from necessity to use high frequencies (in the range of tens of $\mathrm{MHz}$ ). At high frequencies attenuation in composite tends to be strong.

Owing to their compound material structure, carbon fibre composites have also complex electromagnetic properties: electrically conductive carbon fibre is embedded into a dielectric resin matrix. Therefore, CFRPs lend themselves to a range of electromagnetic non-destructive techniques: eddy current testing (ECT), capacitive sensing, and inductive thermography. These techniques are complementary to the traditional UT since they enable NDT of very thin layers and can be applied to dry, wet and consolidated carbon based materials [20]. They offer possibility of imaging (either using arrays of inductors/electrodes or a thermal camera) and can also be easily automated/robotised. Since carbon is a reasonably good electrical conductor, ECT is 
suitable for inspection of integrity of fibres and their quality (fibre density, orientation, undulation etc.) [21-25]. Importantly, electrical conductivity of unidirectional single layered CFRP is anisotropic and is reported to be approximately $5 \mathrm{MS} / \mathrm{m}$ in the longitudinal direction and $1 \mathrm{kS} / \mathrm{m}$ in the transversal direction [22]. An NDT system that relates eddy-current sensor responses to the fibre layup of a composite structure, the presence of impact damage on a composite structure with or without a metal liner, volumetric stress within the composite and fibre tow density has been patented [26]. Due to the relatively low electrical conductivity of carbon fibre, inductors used for ECT of composites operate at high excitation frequencies from $100 \mathrm{kHz}$ to $100 \mathrm{MHz}[20,21]$. Therefore, beside the eddy currents, displacement currents become very pronounced and thus capacitance effects become pronounced.The reported systems can frequently determine material properties related to the presence of buried defects in the dielectric phase of composite materials (resin), such as moisture ingress, aging of the material due to service or environmental/thermal exposure, voids and delaminations [26]. However, it has been noted that delaminations in CFRP cannot be detected by means of ECT because the eddy currents flow parallel to the surface, unless there are inter-laminar fibre contacts [27]. Capacitive Imaging (CI), which is based on the variation of dielectric properties of the resin matrix, enables detection of delaminations caused by impact damage [28]. There has been extensive research in the field of capacitive imaging of concrete structures [29, 30] and glass fibre composites [31, 32] by means of coplanar contiguous electrodes. A concentric coplanar capacitive sensor including a charged central disc forming a first electrode, an outer annular ring coplanar with and outer to the charged central disc, and a method providing for determining transcapacitance between the first electrode and the second electrode using the transcapacitance in a model that accounts for a dielectric test piece to inversely determine the properties of the dielectric test piece has been patented [33]. However, 
there are very few publications regarding capacitive imaging of CFRP, mainly of surface features at excitation frequencies from $10 \mathrm{kHz}$ to $1 \mathrm{MHz}$ [34].

A limitation is believed to be due to electrical conductivity of carbon fibre which produces electromagnetic shielding since the electric field lines are attracted to the conductive fibres and result in surface electric currents. Since electrical conductivity is anisotropic, orientating coplanar electrodes so that the injected electric field is perpendicular to fibres will reduce this shielding effect. Another effect consists in the fact that in similar way as high frequency ECT generate considerable displacement currents, alternating current $\mathrm{CI}$ will induce alternating magnetic field in the CFRP which in turn will generate eddy currents in the carbon fibre. Therefore CI can be sensitive not only to defects in the dielectric matrix, but also to defects of fibre. This work presents a demonstration of capacitive non-destructive imaging of impact damage in woven fibre CFRP using a coplanar capacitive sensor. Spectral characteristics of the capacitive sensor response to CFRP properties and choice of excitation frequency for optimum imaging of delamination are discussed. This approach represents improvement of CI of composite materials.

\section{Samples and Experimental Setup}

\subsection{Samples}

Samples represented square slabs of woven fabric CFRP with side of $100 \mathrm{~mm}$ and thickness of 10mm. Each sample had 25 layers of twill weave fabric with alternating orientation. Three of the samples had a polymer film applied to the impact surfaces. Figure 1 shows photographs of the CFRP samples taken from the impact side. Table 1 shows impact energies for each sample. A woven fabric CFRP sample with drilled holes of $6 \mathrm{~mm}$ diameter has been capacitively imaged for reference. Comparison of capacitive 
images of drilled holes with those of impact damage demonstrated that CI of the latter

reveals bigger area due to the subsurface damage than due to the visible impact dent.

\subsection{Ultrasonic Phased Array Setup}

Reference NDT scans of the samples were carried out using an ultrasonic phased array (PA) instrument, Olympus Omni-Scan MX2 and a wheel PA probe RollerFORM (central frequency $5 \mathrm{MHz}, 64$ elements, element pitch $0.8 \mathrm{~mm}$ ). Samples were scanned using an XYZ CNN scanner from the surface opposite to the impact.

\subsection{Capacitive Imaging Setup}

The capacitive probe consisted of two coplanar rectangular electrodes parallel to the sample surface, as shown in Figure 2. An impedance analyser Agilent 4395A was used to measure impedance variation (series resistance and series capacitance) of the coplanar capacitor in the range of swept excitation frequencies from $100 \mathrm{kHz}$ to 500MHz. The CI probe was moved above sample surface in a stepwise motion utilising a XYZ scanner. The scanned are was $70 \mathrm{~mm} \times 70 \mathrm{~mm}$. The steps were $1 \mathrm{~mm}$ in $\mathrm{X}$ and $2 \mathrm{~mm}$ in $\mathrm{Y}$ directions.

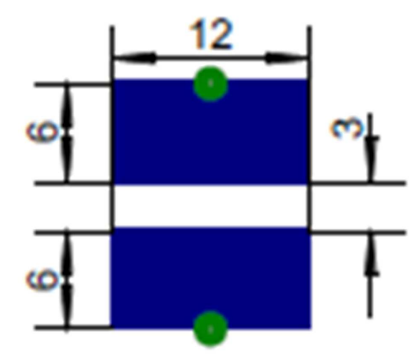

Figure 2. Drawing of the capacitive probe 


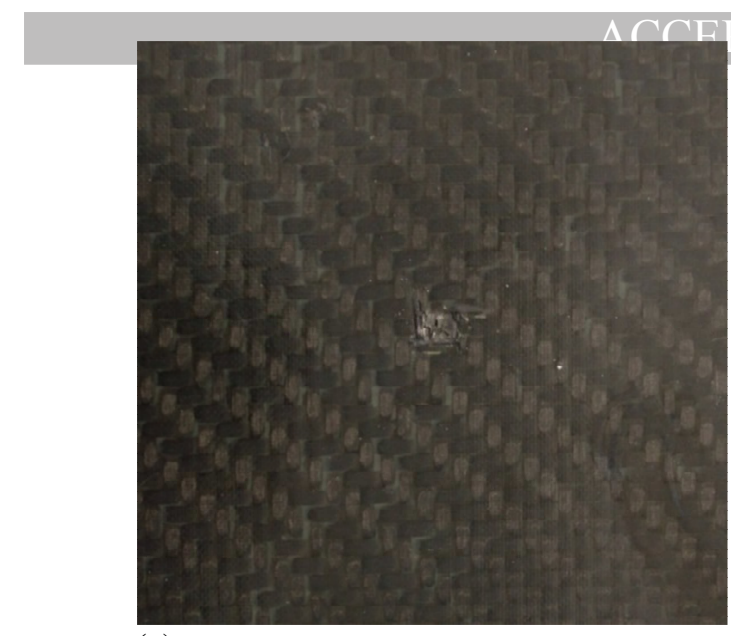

(a)

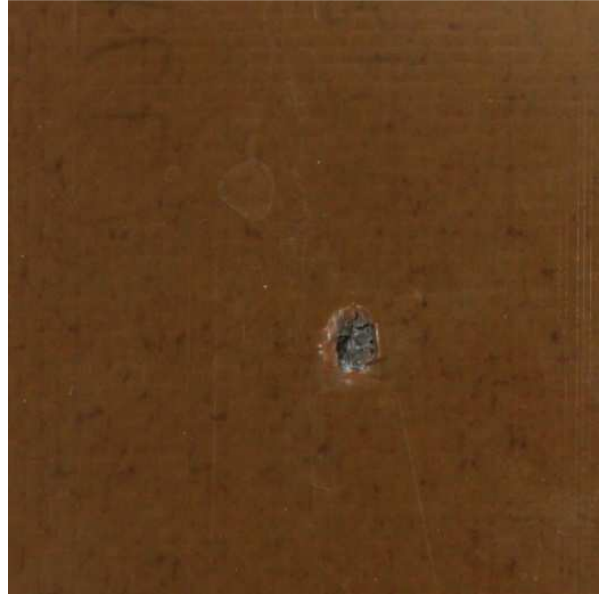

(c)

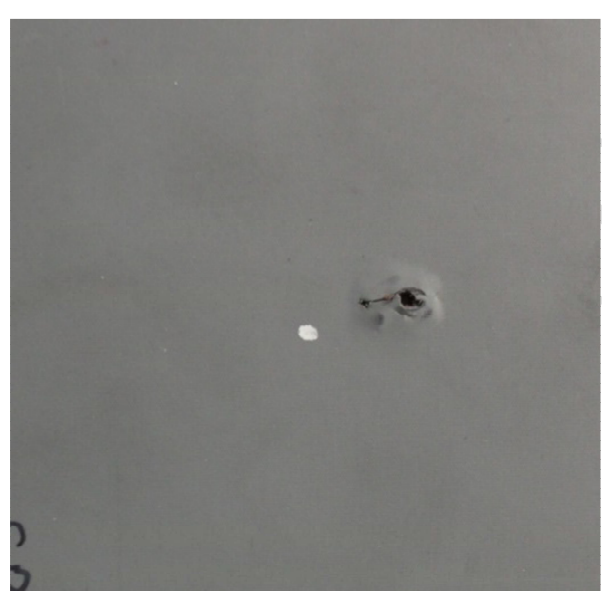

(e)

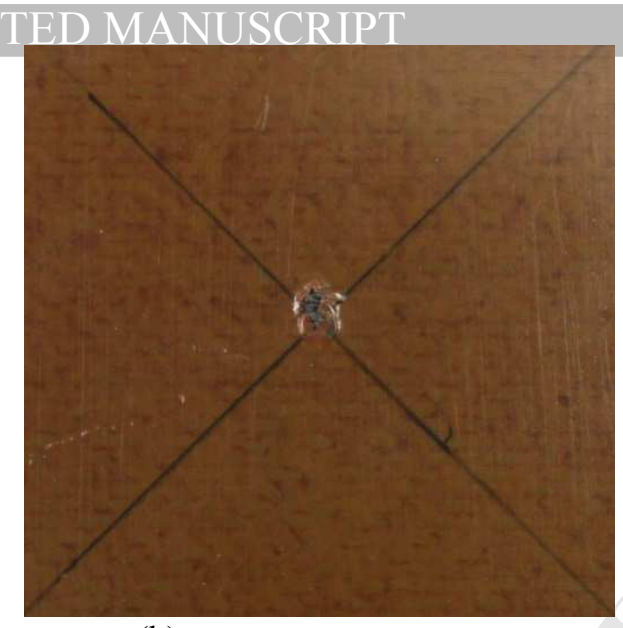

(b)

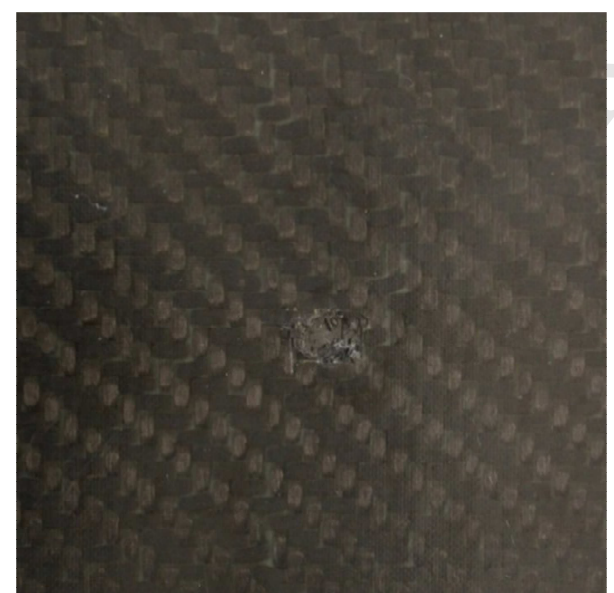

(d)

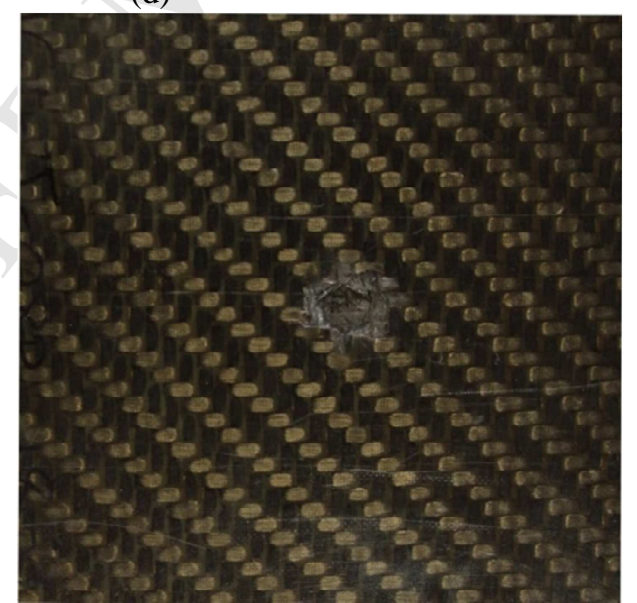

(f)

Figure 1. Photographs of the CFRP samples, impact side: (a) IG4J-08A, (b) IG4Q-04H, (c) IG4Q-04N, (d) IG4J-08G, (e) IG4A-01C, (f) IG4J-08B

\section{Results and discussion}



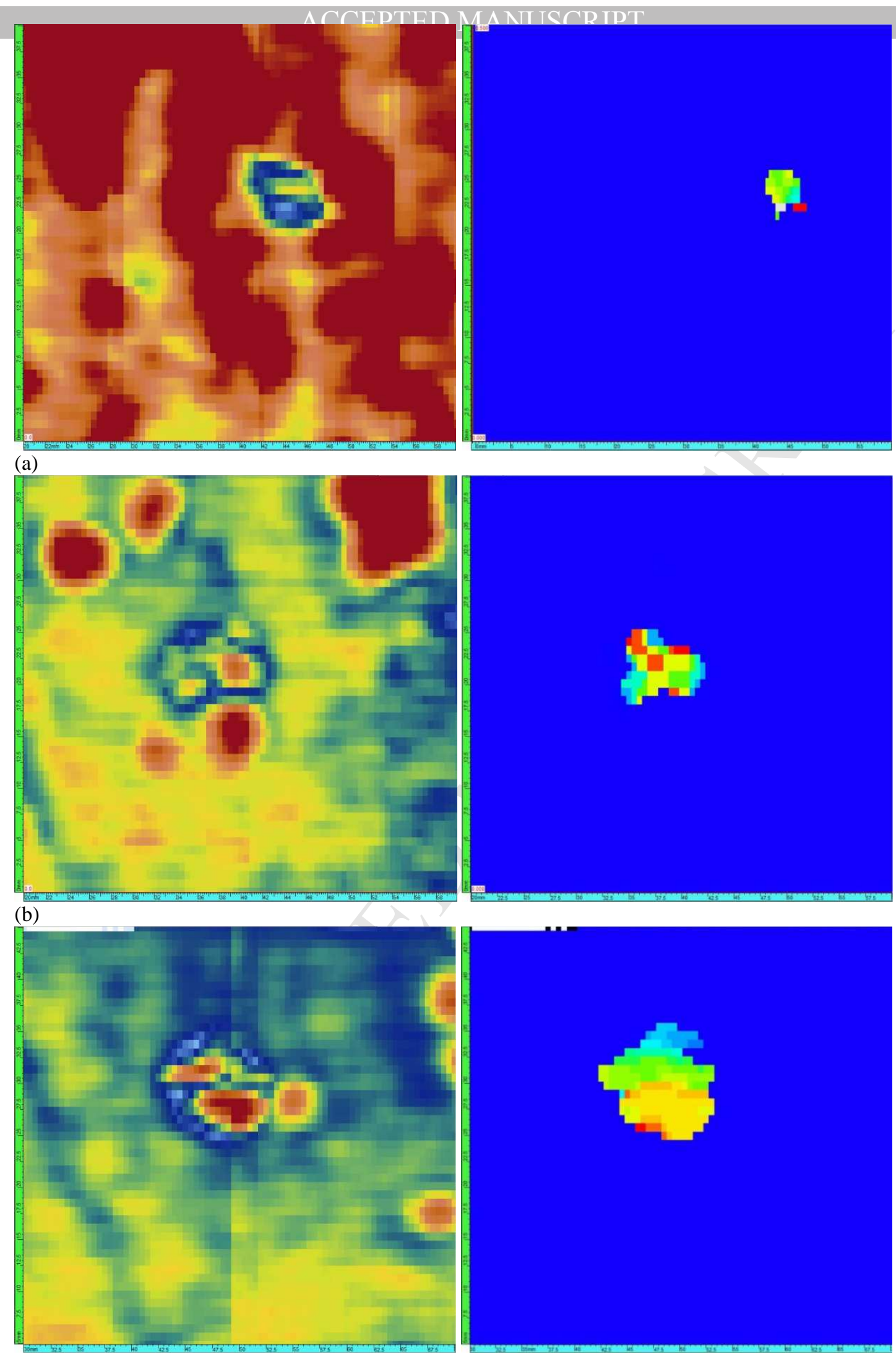

(c)

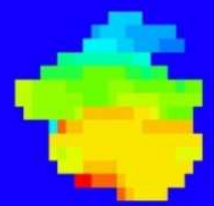



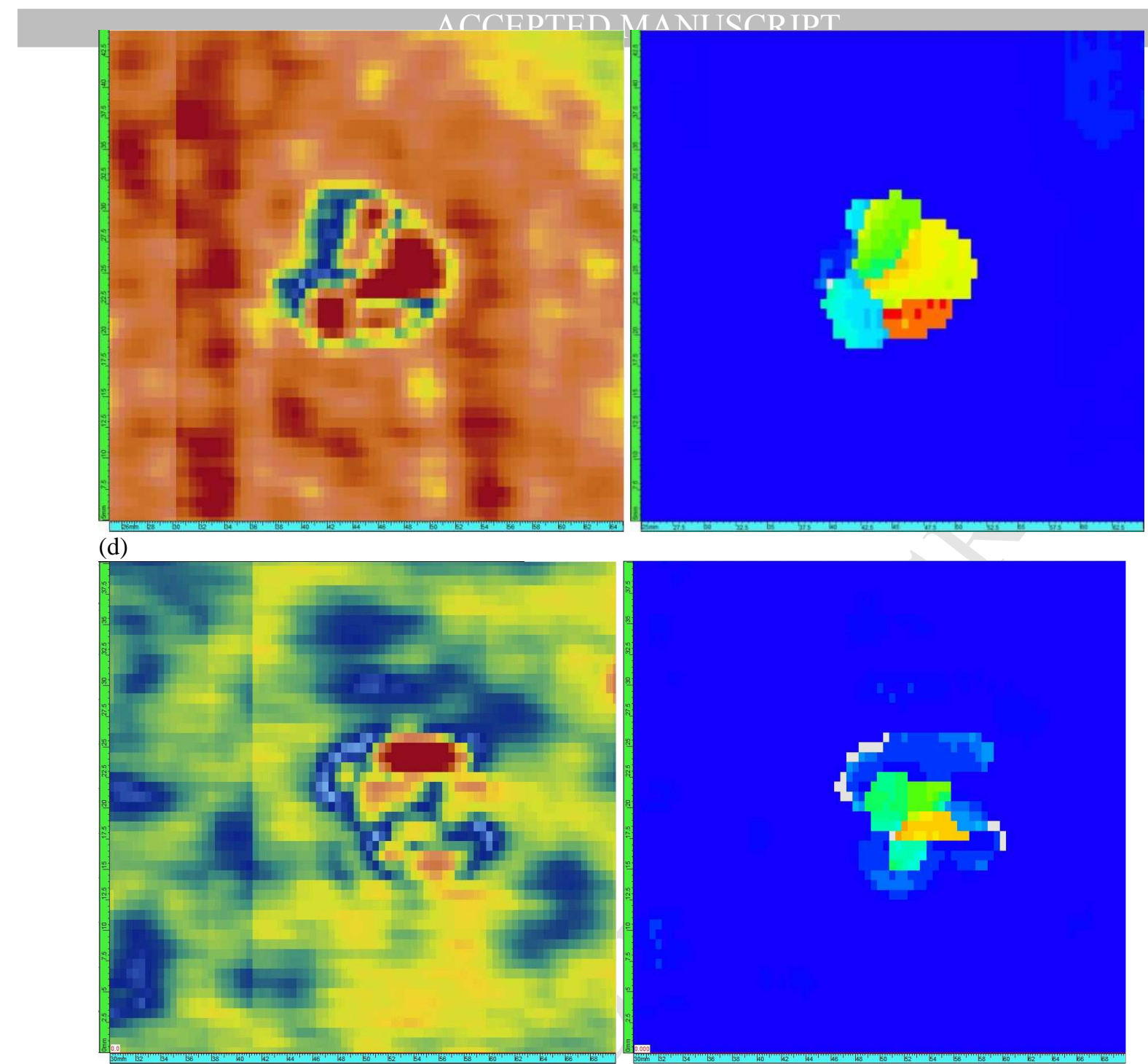

(e)
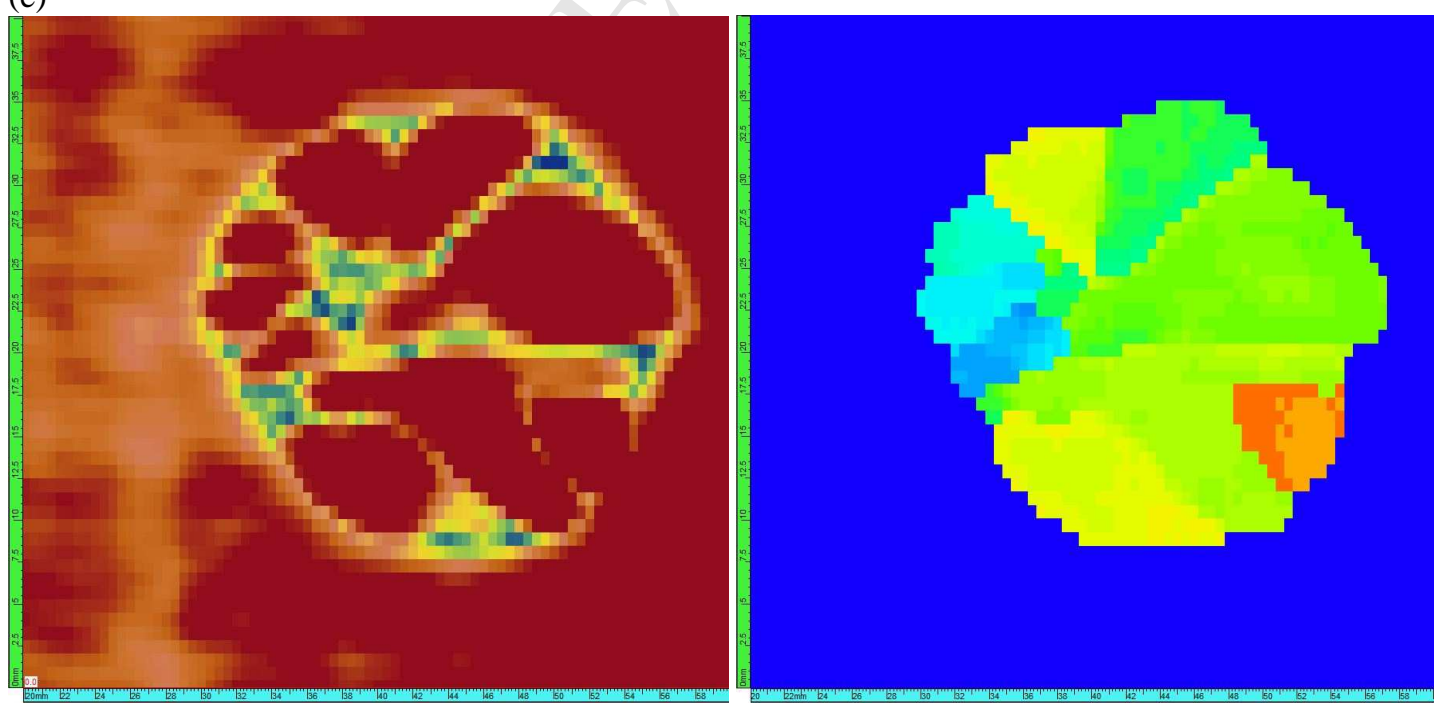

(f)

Figure 3. Ultrasonic C-scans (40mm X 40mm) of delamination due to impact in the CFRP samples, left column represents peak amplitude, right column represents thickness: (a) IG4J-08A, (b) IG4Q-04H, (c) IG4Q-04N, (d) IG4J-08G, (e) IG4A-01C, (f) IG4J-08B 
Figure 3 shows ultrasonic C-scans of delaminations due to impacts in the CFRP samples acquired from the reverse sides of the samples. Areas of the C-scans are $40 \mathrm{~mm} \mathrm{X}$ 40mm. Left column represents peak amplitude and right column represents thickness Cscans. The delamination area grows with increasing impact energy.

Notice that samples (b), (c) and (e) which had polymer films on the impact surfaces (see Fig. 1) have peak amplitude backgrounds which differ from the rest of the samples due to reduced acoustic impedance on the interfaces with the films.

Responses of the CI transducer are obtained in form of a capacitor impedance $\underline{Z}=R_{s}+$ $1 / j \omega C_{s}$, where $R_{s}$ is the series resistance (real part), $\omega=2 \pi f, f$ being the excitation frequency, $C_{s}$ is the series capacitance. Figures $4(\mathrm{a} \& \mathrm{~b})$ show frequency responses obtained in an undamaged location (background) and in the centre (impact) of sample IG4J-08B: serial resistance and serial capacitance, respectively. As expected, capacitance between the coplanar electrodes increases with approach of a dielectric material. Resistance also increases due to eddy currents losses in the conductive carbon fibre. Multiple resonances can be observed between $100 \mathrm{kHz}$ and $200 \mathrm{MHz}$. Certainly, the real electric circuit of the coplanar capacitor electromagnetically coupled with the CFRP material is much more complex than the simplified lumped component model consisting of series resistance and series capacitance assumed by the impedance analyser. Moreover, such a model depends on simplifying assumptions appropriate for a particular excitation frequency. The material structure of CFRP is very complex from the electromagnetic point of view [20,22], carbon fibres insulated by resin represent a complex network of capacitances. Electrical contact between woven fibres effectively represents a network of mesoscopic coils in which eddy currents are induced. Moreover, taking into account that depth of penetration of electromagnetic field into the sample decreases with increasing frequency, it is obvious that at lower frequencies the capacitive transducer is electromagnetically coupled with thicker layer of CFRP. 
However, evaluating the skin depth in CFRP is very challenging due to its inhomogeneous and anisotropic properties. Spectral analysis of CI response is a simple experimental way to determine appropriate excitation frequency of inspection of given material. Based on frequency responses shown in Figure 4 it can be concluded that stable capacitive images can be obtained above $200 \mathrm{MHz}$, since capacitive images at or near resonance frequencies offer low sensitivity/resolution.

Figures 5(a-f) show capacitive images of delaminations caused by impact in the CFRP samples acquired at $300.04 \mathrm{MHz}$, the left column representing series resistance and the right column representing series capacitance. For each sample a clear image of damage is obtained with the area of the defect response being much bigger than area of the visible flaw on the samples' surfaces caused by impact. The sizes of the impact damage signatures in the capacitive images are comparable with those shown in ultrasonic PA C-scans, Fig.3. This indicates that capacitive images of the impacted samples show signatures of subsurface defects, that is the delaminations. It has to be pointed out that the edges of impact dents were flat for the most of the samples, therefore the CI responses are not due to tilting of the transducer. Notice that samples (b), (c) and (e) which had polymer films on the impact surfaces (see Fig. 1) have background signals which differ from the rest of the samples due to different dielectric properties on the interfaces with the films.

Figures $6(\mathrm{a} \& \mathrm{~b})$ show spectrograms obtained along a single line scan in $\mathrm{X}$ direction across sample IG4J-08B at $\mathrm{Y}=35 \mathrm{~mm}$ (B-scans): relative difference serial resistance and relative difference serial capacitance, respectively. A spectrogram is a $2 \mathrm{D}$ representation of the spectrum of frequencies in the CI response as they vary with the scan distance across a sample. Top of a spectrogram (highest frequency) corresponds to the sample surface due to the skin depth. Figures $7(\mathrm{a} \& \mathrm{~b})$ illustrate line scans obtained along a single line scan in $\mathrm{X}$ direction across sample IG4J-08B at $\mathrm{Y}=35 \mathrm{~mm}$ at three 
selected frequencies: absolute difference serial resistance and absolute difference serial capacitance, respectively.

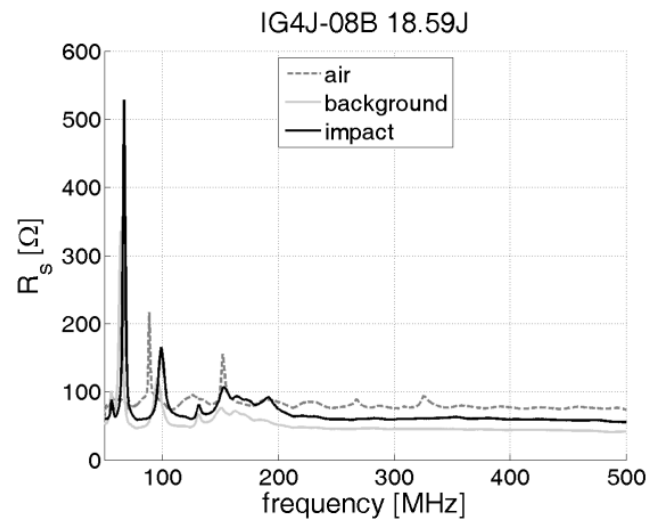

(a)

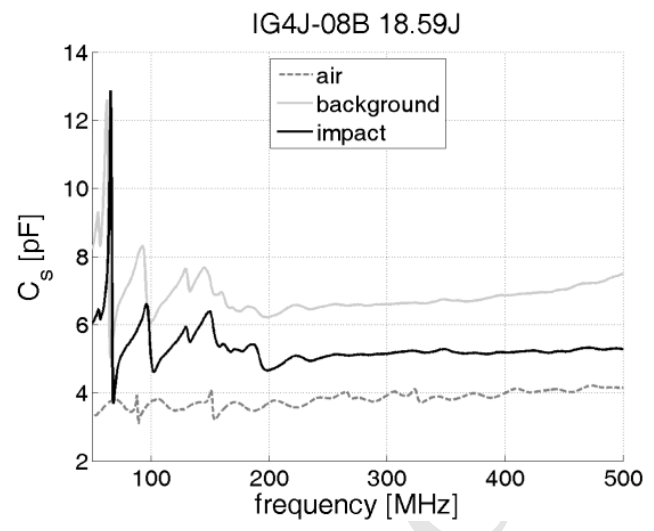

(b)

Figure 4. Frequency responses obtained in an undamaged location (background) and in the centre (impact) of sample IG4J-08B: (a) serial resistance, (b) serial capacitance

(a)
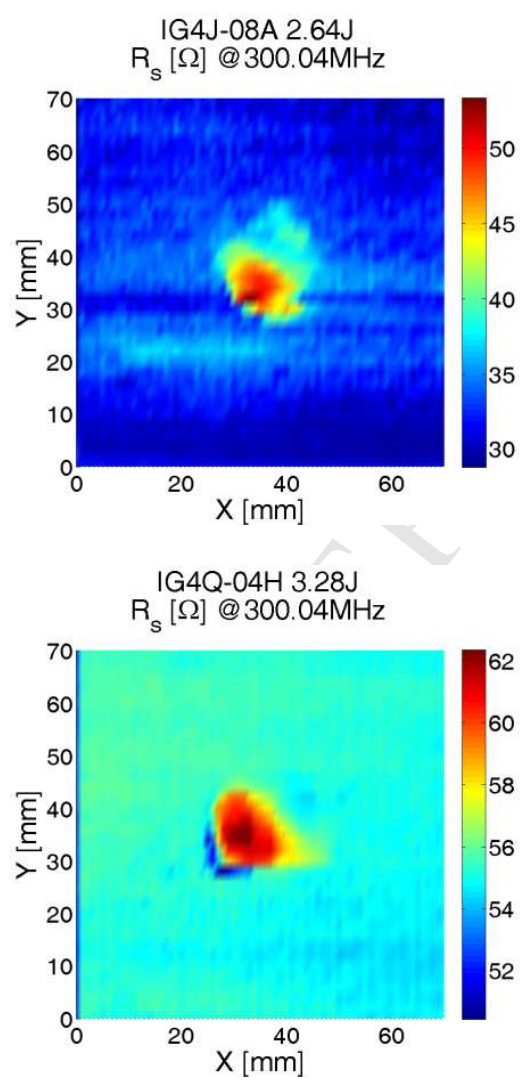

(b)
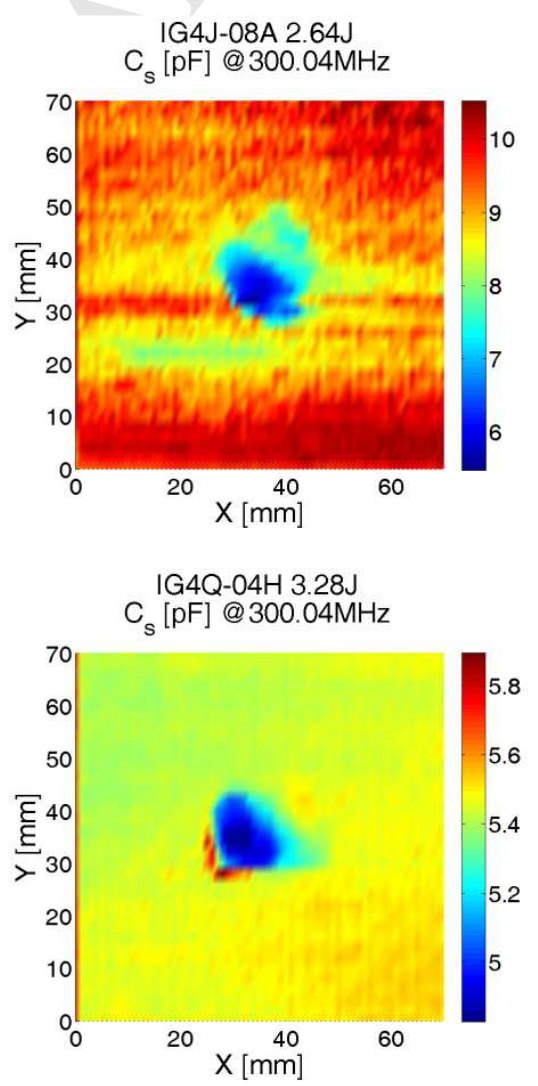


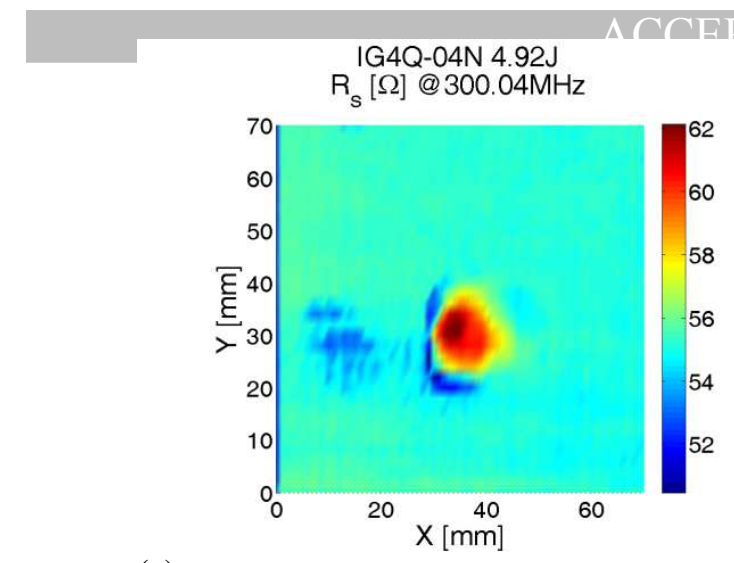

(c)

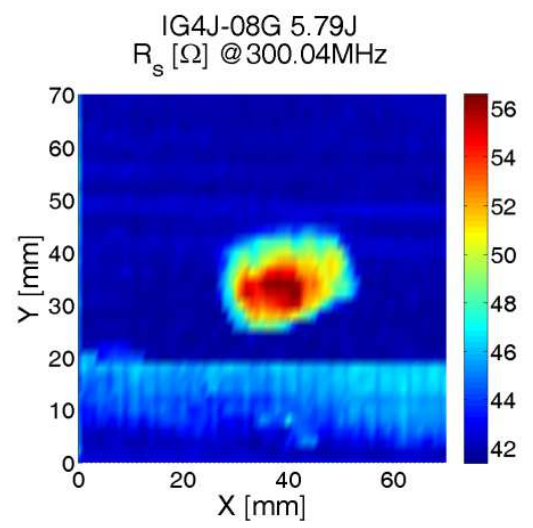

(d)

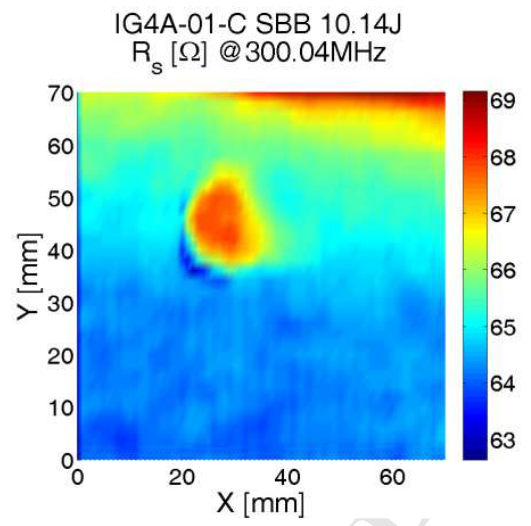

(e)
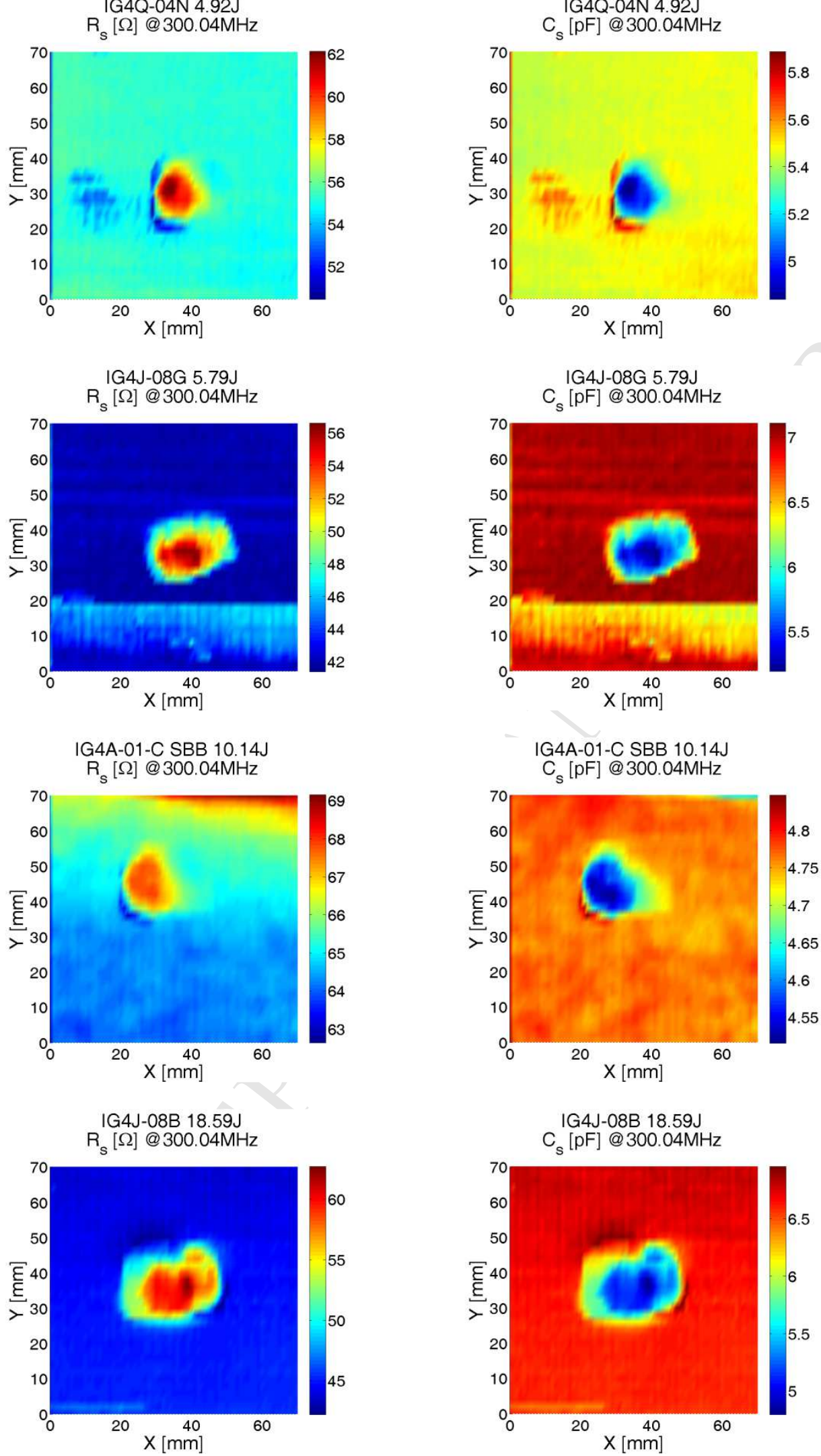

(f)

Figure 5. Capacitive images of delamination due to impact in the CFRP samples acquired at $300.04 \mathrm{MHz}$,

left column represents serial resistance, right column represents serial capacitance: (a) IG4J-08A, (b)

IG4Q-04H, (c) IG4Q-04N, (d) IG4J-08G, (e) IG4A-01C, (f) IG4J-08B 


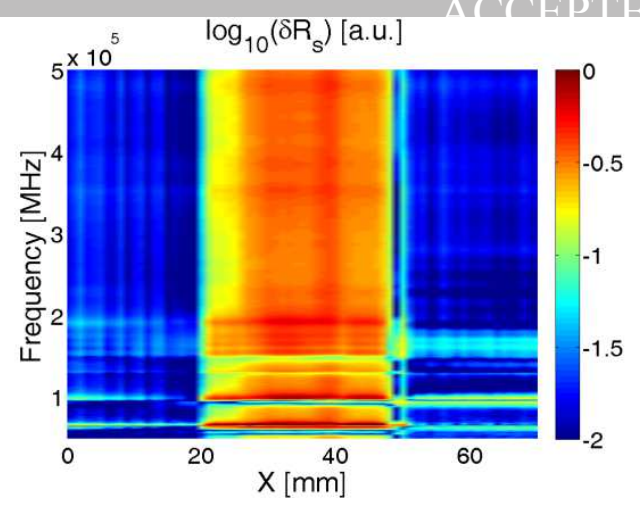

(a)

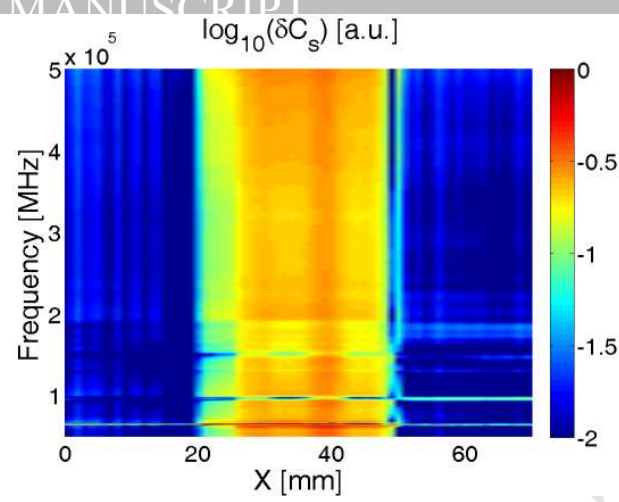

(b)

Figure 6. Spectrograms obtained along a single line scan in $\mathrm{X}$ direction across sample IG4J-08B at $\mathrm{Y}=$ $35 \mathrm{~mm}$ (B-scans): (a) relative difference serial resistance, (b) relative difference serial capacitance
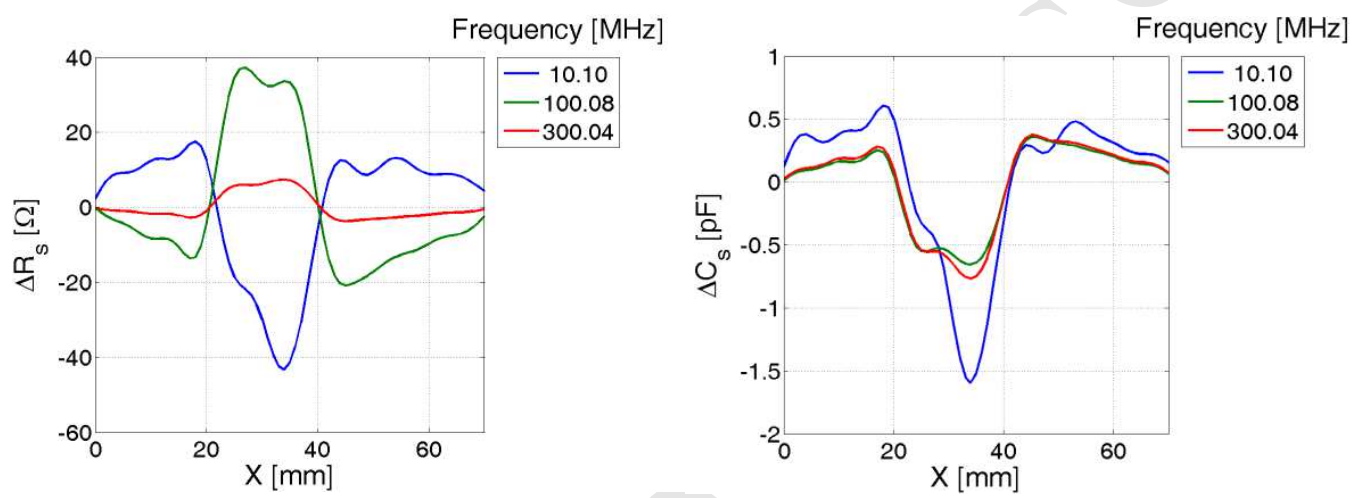

Figure 7. Line scans obtained along a single line scan in $\mathrm{X}$ direction across sample IG4J-08B at $\mathrm{Y}=$ $35 \mathrm{~mm}$ at three selected frequencies: (a) absolute difference serial resistance, (b) absolute difference serial capacitance

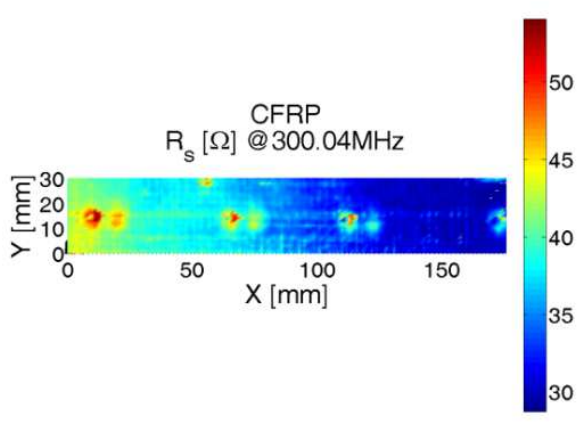

(a)

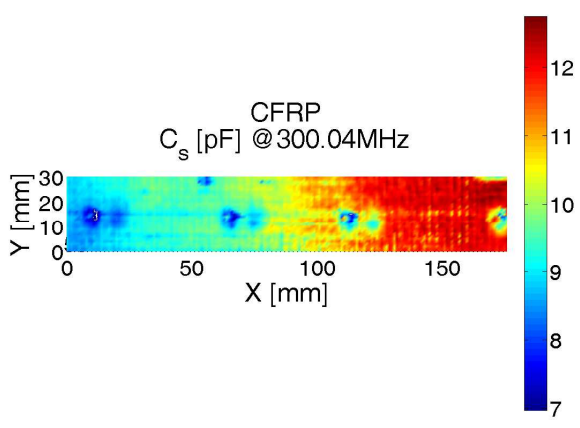

(b)

Figure 8. Capacitive images of drilled holes in a CFRP slab: (a) serial resistance, (b) serial capacitance 
Figures $8(\mathrm{a} \& \mathrm{~b})$ show capacitive images of drilled holes in a CFRP slab: serial resistance and serial capacitance, respectively. The holes had diameters of $6 \mathrm{~mm}$, which roughly correspond to the size of the biggest impact dent in Figure 1(f). The CI signatures of the drilled holes have diameter of circa $6 \mathrm{~mm}$ at half height. This confirms conclusion that capacitive images of the impacted samples show signatures of subsurface defects, that is delaminations.

\section{Conclusions}

This work presented capacitive non-destructive imaging of impact damage in woven fibre CFRP using a coplanar capacitive sensor. Novelty of the approach consists in that spectral characteristics of the capacitive sensor response to CFRP properties were firstly used to establish excitation frequencies for optimum imaging of delamination. For the tested samples optimum excitation frequencies were found to be above $200 \mathrm{MHz}$. Clear capacitive images of delaminations were obtained on each sample. Ultrasonic C-scans of delaminations due to impacts were acquired using phased array technique to confirm presence and extent of the delaminations. CI technique can be used as complementary to UT for imaging shallow defects in composite materials at depths where UT has insufficient time domain resolution.

Further work should address modelling of interaction of electromagnetic field with CFRP composite material and comprehensive analysis of the resulting equivalent electrical circuit in order to optimise sensitivity and resolution of CI transducers.

\section{Acknowledgements}

This research did not receive any specific grant from funding agencies in the public, commercial, or not-for-profit sectors. 
1. Grady, J.E., Impact Damage in Composite Laminates, 1988.

2. Chung-Yue, W. and C.H. Yew, Impact damage in composite laminates. Computers \& Structures, 1990. 37(6): p. 967-982.

3. Richardson, M.O.W. and M.J. Wisheart, Review of low-velocity impact properties of composite materials. Composites Part A: Applied Science and Manufacturing, 1996. 27(12 PART A): p. 1123-1133.

4. Adams, R.D. and P. Cawley, A review of defect types and nondestructive testing techniques for composites and bonded joints. NDT International, 1988. 21(4): p. 208222.

5. Suemasu, H., Impact Damage in Composite Laminates, in POLYMER COMPOSITES From Nano- to Macro-Scale, K. Friedrich, S. Fakirov, and Z. Zhang, Editors. 2005, Springer Science. p. 289-307.

6. Liu, D., Impact-Induced Delamination-A View of Bending Stiffness Mismatching. Journal of Composite Materials, 1988. 22(7): p. 674-692.

7. Sultan, M.T.H., et al., On impact damage detection and quantification for CFRP laminates using structural response data only. Mechanical Systems and Signal Processing, 2011. 25(8): p. 3135-3152.

8. Gaudenzi, P., et al., On the evaluation of impact damage on composite materials by comparing different NDI techniques. Composite Structures, 2014. 118(1): p. 257-266.

9. Karbhari, V., ed. Non-Destructive Evaluation (NDE) of Polymer Matrix Composites. 2013, Woodhead Publishing. 716.

10. Katunin, A., K. Dragan, and M. Dziendzikowski, Damage identification in aircraft composite structures: A case study using various non-destructive testing techniques. Composite Structures, 2015. 127: p. 1-9.

11. Dong, J., et al., Nondestructive evaluation of forced delamination in glass fiberreinforced composites by terahertz and ultrasonic waves. Composites Part B: Engineering, 2015. 79: p. 667-675.

12. Meola, C., et al., Nondestructive evaluation of carbon fibre reinforced composites with infrared thermography and ultrasonics. Composite Structures, 2015. 134: p. 845-853.

13. Sohn, H., et al., Delamination detection in composites through guided wave field image processing. Composites Science and Technology, 2011. 71(9): p. 1250-1256.

14. Flynn, E.B., et al., Imaging and characterizing structural defects through the estimation of local dispersion curves, in Key Engineering Materials2013. p. 956-961.

15. Rogge, M.D. and C.A.C. Leckey, Characterization of impact damage in composite laminates using guided wavefield imaging and local wavenumber domain analysis. Ultrasonics, 2013. 53(7): p. 1217-1226.

16. Leckey, C.A.C., M.D. Rogge, and F. Raymond Parker, Guided waves in anisotropic and quasi-isotropic aerospace composites: Three-dimensional simulation and experiment. Ultrasonics, 2014. 54(1): p. 385-394.

17. Manson, G., et al., Some experimental observations on the detection of composite damage using lamb waves. Strain, 2011. 47(SUPPL. 1): p. e254-e268.

18. Park, B., Y.K. An, and H. Sohn, Visualization of hidden delamination and debonding in composites through noncontact laser ultrasonic scanning. Composites Science and Technology, 2014. 100: p. 10-18.

19. Karabutov, A.A. and N.B. Podymova, Quantitative analysis of the influence of voids and delaminations on acoustic attenuation in CFRP composites by the laser-ultrasonic spectroscopy method. Composites Part B: Engineering, 2014. 56: p. 238-244.

20. Heuer, H., et al. Process monitoring for resins, carbon fiber fabrics, preforms and consolidated CFRPS by HF radio wave techniques. in 54th Annual British Conference of Non-Destructive Testing, NDT 2015. 2015. 
21. Heuer, H., M. Schulze, and R. Heere. Non-destructive flaw detection in in-situ carbon fibre reinforced composites using eddy-current. in Proceedings of the 7th International Conference on FRP Composites in Civil Engineering, CICE 2014. 2014.

22. Heuer, H., M.H. Schulze, and N. Meyendorf, Non-destructive evaluation (NDE) of composites: Eddy current techniques, in Non-Destructive Evaluation (NDE) of Polymer Matrix Composites: Techniques and Applications2013. p. 33-55.

23. Mizukami, K., et al., Detection of in-plane fiber waviness in cross-ply CFRP laminates using layer selectable eddy current method. Composites Part A: Applied Science and Manufacturing, 2016. 82: p. 108-118.

24. Mizukami, K., et al., Detection of in-plane and out-of-plane fiber waviness in unidirectional carbon fiber reinforced composites using eddy current testing. Composites Part B: Engineering, 2016. 86: p. 84-94.

25. Salski, B., et al., Non-destructive testing of carbon-fibre-reinforced polymer materials with a radio-frequency inductive sensor. Composite Structures, 2015. 122: p. 104-112.

26. Goldfine, N.J., et al., Method and apparatus for non-destructive evaluation of materials, 2015: USA. p. 25.

27. Mook, G., R. Lange, and O. Koeser, Non-destructive characterisation of carbon-fibrereinforced plastics by means of eddy-currents. Composites Science and Technology, 2001. 61(6): p. 865-873.

28. Diamond, G.G., D.A. Hutchins, and T.H. Gan. Defect location using capacitative imaging. in AIP Conference Proceedings. 2008.

29. Sharma, D., et al., Non-destructive testing of materials using capacitive sensing technique. MIT International Journal of Electronics and Communication Engineering, 2011. 1(2): p. 73-77.

30. Yin, X., et al., Non-destructive evaluation of concrete using a capacitive imaging technique: Preliminary modelling and experiments. Cement and Concrete Research, 2010. 40(12): p. 1734-1743.

31. Asmatulu, R., B. Venishetty, and E. Asmatulu. Non-destructive testing of fiber reinforced composite materials using a capacitance bridge. in ASME International Mechanical Engineering Congress and Exposition, Proceedings. 2010.

32. Yin, X., et al., Preliminary studies on the design principles of capacitive imaging probes for non-destructive evaluation. International Journal of Applied Electromagnetics and Mechanics, 2013. 42(3): p. 447-470.

33. Bowler, N. and T. Chen, Concentric coplanar capacitive sensor for nondestructive evaluation, 2012, lowa state university research foundation: USA.

34. Yin, X. and D.A. Hutchins, Non-destructive evaluation of composite materials using a capacitive imaging technique. Composites Part B: Engineering, 2012. 43(3): p. 12821292. 


\section{Figure Captions}

Figure 1. Photographs of the CFRP samples, impact side: (a) IG4J-08A, (b) IG4Q-04H,

(c) IG4Q-04N, (d) IG4J-08G, (e) IG4A-01C, (f) IG4J-08B

Figure 2. Drawing of the capacitive probe

Figure 3. Ultrasonic C-scans (40mm X 40mm) of delamination due to impact in the CFRP samples, left column represents peak amplitude, right column represents thickness: (a) IG4J-08A, (b) IG4Q-04H, (c) IG4Q-04N, (d) IG4J-08G, (e) IG4A-01C, (f) IG4J-08B

Figure 4. Frequency responses obtained in an undamaged location (background) and in the centre (impact) of sample IG4J-08B: (a) serial resistance, (b) serial capacitance Figure 5. Capacitive images of delamination due to impact in the CFRP samples acquired at $300.04 \mathrm{MHz}$, left column represents serial resistance, right column represents serial capacitance: (a) IG4J-08A, (b) IG4Q-04H, (c) IG4Q-04N, (d) IG4J-08G, (e) IG4A-01C, (f) IG4J-08B

Figure 6. Spectrograms obtained along a single line scan in X direction across sample IG4J-08B at $\mathrm{Y}=35 \mathrm{~mm}$ (B-scans): (a) relative difference serial resistance, (b) relative difference serial capacitance Figure 7. Line scans obtained along a single line scan in X direction across sample IG4J-08B at $\mathrm{Y}=35 \mathrm{~mm}$ at three selected frequencies: (a) absolute difference serial resistance, (b) absolute difference serial capacitance Figure 8. Capacitive images of drilled holes in a CFRP slab: (a) serial resistance, (b) serial capacitance 
Tables

Table 1. Impact energies

\begin{tabular}{lllllll}
\hline Sample ID & IG4J-08A & IG4Q-04H & IG4Q-04N & IG4J-08G & IG4A-01C & IG4J-08B \\
\hline Impact energy (J) & 2.64 & 3.28 & 4.92 & 5.79 & 10.14 & 18.59 \\
\hline
\end{tabular}

